\title{
Human cooperation by lethal group competition
}

SUBJECT AREAS:

BEHAVIOURAL ECOLOGY

SOCIAL EVOLUTION

ANIMAL BEHAVIOUR

PSYCHOLOGY

Received

21 February 2012

Accepted

18 February 2013

Published

5 March 2013

Correspondence and requests for materials should be addressed to

M.E. (Egas@uva.nl)

\author{
Martiin Egas ${ }^{1}$, Ralph Kats ${ }^{1}$, Xander van der Sar ${ }^{1}$, Ernesto Reuben² \& Maurice W. Sabelis ${ }^{1}$
}

'Institute for Biodiversity and Ecosystem Dynamics, Section Population Biology, University of Amsterdam, Science Park 904, P.O.Box 94248, 1090 GE Amsterdam, The Netherlands, ${ }^{2}$ Management Division, Columbia Business School, Columbia University, 705 Uris Hall, 3022 Broadway, New York, NY 10027, USA.

Why humans are prone to cooperate puzzles biologists, psychologists and economists alike. Between-group conflict has been hypothesized to drive within-group cooperation. However, such conflicts did not have lasting effects in laboratory experiments, because they were about luxury goods, not needed for survival ("looting"). Here, we find within-group cooperation to last when between-group conflict is implemented as "all-out war" (eliminating the weakest groups). Human subjects invested in helping group members to avoid having the lowest collective pay-off, whereas they failed to cooperate in control treatments with random group elimination or with no subdivision in groups. When the game was repeated, experience was found to promote helping. Thus, not within-group interactions alone, not random group elimination, but pay-off-dependent group elimination was found to drive within-group cooperation in our experiment. We suggest that some forms of human cooperation are maintained by multi-level selection: reciprocity within groups and lethal competition among groups acting together.

T he evolution and maintenance of cooperation among unrelated individuals is a fundamental problem in the biological and social sciences. Theoretical conditions favouring cooperation include genetic relatedness among cooperators, situations allowing for direct benefits to the cooperator, and repeated interactions allowing for indirect benefits to the cooperator via reciprocal altruism and reputation building ${ }^{1,2}$. In absence of these conditions, however, humans show more cooperation than theory predicts. Group structure may be a factor bridging the gap between theory and experiment, but as yet its role in coooperation is a highly debated topic ${ }^{3-7}$. Recent theory points to a role for inter-group conflicts ${ }^{8-12}$. The idea is that individuals are engaged in a conflict between personal performance and group performance, causing the emerging levels of cooperation to be a compromise. This is the modern way to view the process of group selection: group structure matters to the extent in which it influences the individual interests.

The question how group conflicts affect decisions of individuals within groups, has received some attention in social psychology ${ }^{13}$, where so-called "team games" are used to identify conditions under which individual or collective rationality prevails. So far, experiments involved non-lethal group competition and failed to show a lasting effect on cooperation ${ }^{13-15}$. If effects on cooperation were demonstrated, then they were the result of aligning personal interest with group benefit ${ }^{16}$. In this article, we design a repeated "team game" experiment that differs in particular by (I) eliminating groups based on group performance criteria (lethal group competition instead of non-lethal as done in experiments published so $\mathrm{far}^{13-16}$ ), by (II) making individuals play multiple rounds of the game instead of one-shot games (as reviewed in $^{13}$ ), and by (III) setting the rule that the ultimate winner of the game is the individual in the surviving group with the highest payoff over all rounds. This design causes group competition to be extreme and the conflict of interest for individuals between group performance and personal performance to be as high as possible. To assess the consequences experimentally we explored three versions of the game differing in the way group elimination was executed: elimination of the group with the lowest pay-off (the sum of individual pay-offs), random elimination of a group, and no elimination. Here, we show experimentally that lethal competition by pay-off-dependent group elimination can drive within-group cooperation in humans.

\section{Results}

Under the treatment where the poorest group was eliminated, group members on average invested a significant fraction of EMUs in other group members (Figure 1a). Only in the two-group stage of the tournament this average fraction decreased. In subsequent games, the per-individual investment in the first round significantly increased (Figure 2). Individuals contributed increasingly more in the first round of the three games $\left(\mathrm{F}_{2,357}=11.5\right.$, 

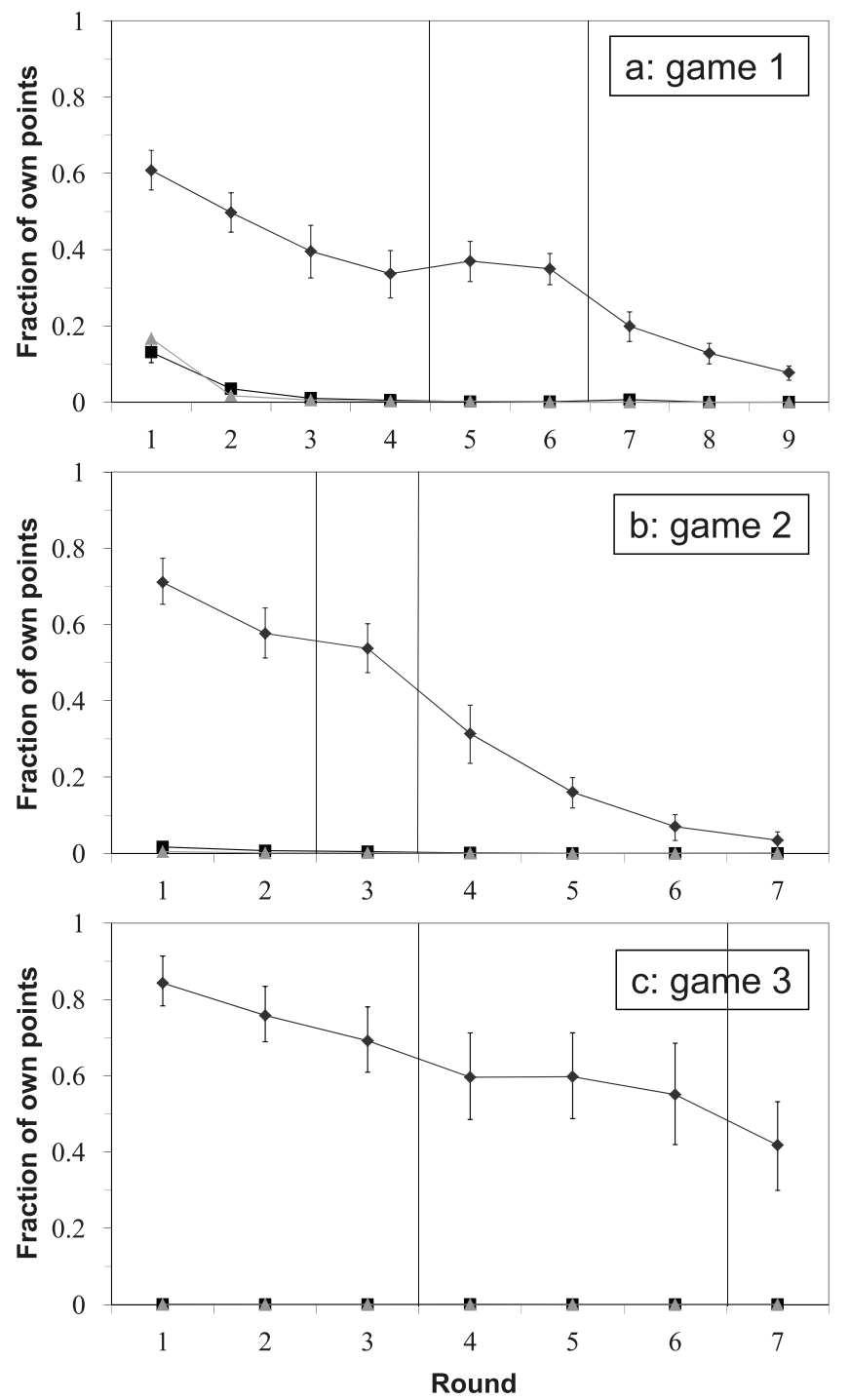

Figure 1 Human cooperation by lethal group competition in the "together alone" "team game". Per-replicate average fraction of EMUs $( \pm \mathrm{SE}$ ) invested by participants in their group members per round of the experiment. All subjects played three (panel a,b,c) games sequentially (group members were reshuffled among groups between games). Vertical lines indicate the moments at which groups were eliminated. In the pay-off-dependent group elimination treatment (diamonds, $\mathrm{N}=10$ ), the poorest group (i.e., with the least EMUs) was eliminated whereas in the group-structured control treatment (squares, $\mathrm{N}=8$ ) the eliminated group was randomly selected. The control treatment (triangles, $\mathrm{N}=7$ ) involved single groups and thus no elimination. Individuals experiencing the pay-off-dependent group elimination treatment invested significant amounts in their group members until two groups remained (after which cooperation broke down). In contrast, individuals in the two control treatments (with and without group structure) did not invest in others at all.

$\mathrm{p}<0.0001)$, but this increase slowed down as participants approached full contribution in game 3 (Figure 1c; Figure 2). This demonstrates a positive effect of learning on cooperative investment.

To control for elimination based on group pay-off, we performed an additional experiment with random group elimination. Assuming rational decision making among all players, game theory predicts that subjects should refrain from investing in others. This prediction is met in our experiments except in the first few rounds (Figure 1a), but this first-round effect vanished in the two subsequent games

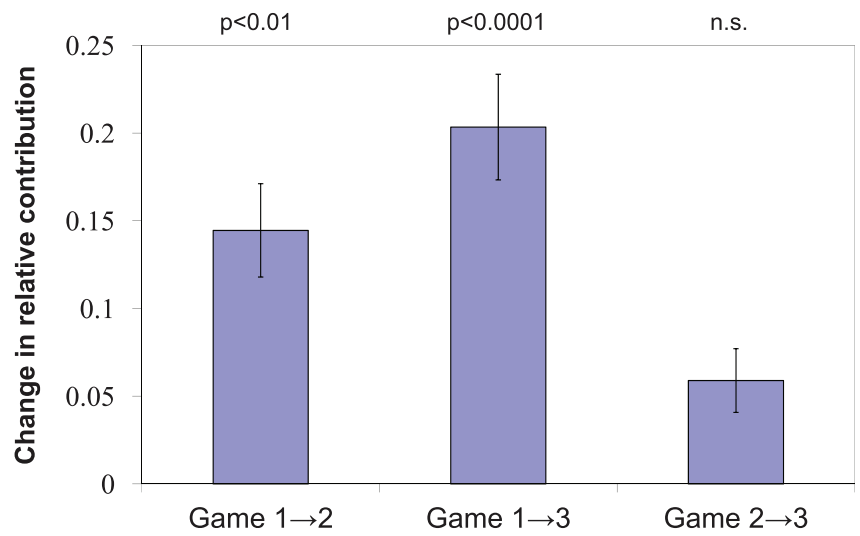

Figure 2 Cooperative investment increased with game experience, as expressed by the average $( \pm \mathrm{SE})$ change in relative per-subject contribution (i.e., fraction of EMUs invested in the other group members) in the first round of each game. This is manifested as a significant increase in relative contribution between games 1 and 2 (left bar; $\mathrm{F}_{1,238}=9.29, \mathrm{p}<0.01$ after Bonferroni correction) and between games 1 and 3 (middle bar; $\mathrm{F}_{1,238}=21.8, \mathrm{p}<0.0001$ after Bonferroni correction), but not between games 2 and 3 (right bar; $\mathrm{F}_{1,238}=2.64$, n.s.).

(Figure 1b,c). To control for group elimination we performed a final experiment in which no elimination occurred. The mode of elimination affected the mean investment for each of the three games (repeated measures ANOVA; Game 1: $\mathrm{F}_{2,22}=70.8, \mathrm{P}<0.0001$; Game 2: $\mathrm{F}_{2,22}=94.1, \mathrm{P}<0.0001$; Game 3: $\mathrm{F}_{2,22}=63.9, \mathrm{P}<$ $0.0001)$.The results of the no-elimination experiment appeared to be statistically indistinguishable from those obtained under random group elimination, (Bonferroni posthoc tests in both comparisons in all three games yielded $\mathrm{p}>0.9$ ) and those of both control experiments were statistically different from the experiments involving pay-off based group elimination (Bonferroni posthoc tests in both comparisons in all three games yielded $\mathrm{p}<0.0001$ ).

Round-to-round changes in the fraction investment may depend on elimination events: (I) the phase before the first elimination, (II) the phase in between the first and second eliminations, and (III) the phase after the second elimination. Such dependencies cannot be detected in the treatments involving random group elimination and no elimination due to the low investment overall. In the treatment with lethal group elimination, however, we found statistical evidence that investments declined significantly after the second elimination event (Table 1) but not after the first (Table 1), except for game 2 where significant declines were found after both elimination events. Also, we observed decreasing investment with rounds in phase I and phase III, but not in phase II (Fig. 1). Comparison of these features across games (Fig. 1a-c) suggests no differences except for a decline in phase I that was less pronounced in the last game than in the previous games.

\section{Discussion}

We found that cooperation only emerged under pay-off-dependent group elimination. Thus, it is not group elimination per se that drives

Table 1 | Paired t-tests of average investment per group for the treatment with lethal group elimination, comparing the round before and after the first elimination event, and the round before and after the second elimination event in each of the three games

\begin{tabular}{lll} 
& First elimination event & Second elimination event \\
\hline Game 1 & $t_{9}=-0.454$, n.s. & $t_{9}=5.38, \mathrm{p}<0.001$ \\
Game 2 & $t_{9}=2.59, \mathrm{p}<0.05$ & $t_{9}=6.76, \mathrm{p}<0.0001$ \\
Game 3 & $t_{9}=2.17$, n.s. & $t_{9}=3.02, \mathrm{p}<0.01$ \\
\hline
\end{tabular}


human cooperation in our experiment. Because we observed no cooperation in the two control treatments, other mechanisms favouring cooperation, such as rewarding or punishment in response to the mean contribution of others in the group, can be ruled out.

We claim that the effect of pay-off-dependent group elimination on cooperation within groups is independent of most details of our together-alone game. Our game differs from the well-known public goods game in that (I) the investor does not receive any return from his own investment, (II) the subjects accumulate EMUs over rounds of the game, and (III) only one group member receives payoff at the end of the game. Whereas rule (III) is implemented to strengthen the conflict of individual interest between the subject and its group, rules (I) and (II) are rooted in biology. There are many examples of cooperation where the individual contributing to a public good does not or cannot benefit from the public good to the same extent as others in the group - in the extreme, individuals may die when performing the cooperative $\mathrm{act}^{17}$. It is also quite common that the condition of individuals depends on the returns from cooperative behaviour such that individuals with high returns have more to invest the next time this behaviour is invoked.

Our experimental results are generally in agreement with Bowles' review $^{12}$ on inter-group conflict and group extinction in humans. Bowles $^{12}$ pointed out that group competition promotes within-group cooperation most strongly when the group members gain in equal measures from outcompeting another group. This element in his theoretical framework, called reproductive levelling, is not present in our game. Our results, however, imply that such reproductive levelling is not a necessary condition for within-group cooperation to emerge, as long as there are more than two groups competing with each other (note that cooperation is high until the two-group stage of the tournament, probably because participants start thinking more about their personal interest of winning the game by cooperating less).

Thus, human cooperation may result from pay-off dependent lethal competition between groups, acting in concert with direct and/or indirect reciprocity between members of a group. Two critical issues remain, however: our experimental setup excluded a choice for group or group members as well as within-group negotiation. If group formation is driven by a preference for within-group norms with respect to punishment and/or reputation building, cooperation is shown to increase in some groups but decrease in others (e.g. Ref. 18). Such differentiation in cooperative behaviour among groups is also expected if negotiation among group members would be allowed $\left(\mathrm{cf}^{19}\right)$. Hence, we hypothesize that preference-driven group formation, within-group negotiation and within-group reciprocity create the variation among groups necessary to make lethal group competition a relevant selection mechanism at the level of the population as a whole.

\section{Methods}

The "together alone" game. Repeated "team games" were played with 12 individuals, placed in 4 groups of 3 subjects each. The subjects remain part of the same group over all rounds of the game. Within the group subjects make decisions in a social dilemma situation. Every subject received an endowment of 100 experimental money units (EMUs). In every round of the game subjects had to decide how much of their EMUs they invest in helping the other (anonymous) subjects in their group. This investment was multiplied by a factor, arbitrarily set to 1.5 , and then equally divided over the other two subjects. EMUs not invested remained in possession of the subject. In each round, the other two subjects in a group made their decision simultaneously. The result of each round of the game (i.e. EMUs not invested plus EMUs received from help by the other two group members) formed the starting point for the next. Thus, each subject accumulated EMUs over all rounds of the game. This game is played for a number of rounds unknown to the subjects. Each subject receives information on total EMUs of its group members at the end of each round, and the winner of the game is the one that has accumulated most EMUs over all rounds.

We introduce pay-off-dependent group elimination by involving four groups in a game, and subsequently eliminating the group with lowest total EMUs (i.e., "your group loses, you die") at the end of three arbitrarily chosen rounds. This treatment is called the group competition treatment. After each round subjects received information about the investments of the other subjects in their group, as well as the total amount of EMUs of each of the other groups. Elimination of the group with the lowest total EMUs was done at the end of a round randomly chosen before the experiments. The subjects were aware that these rounds were randomly chosen, and did not know when they would take place. In the event that multiple groups had the same and lowest total, one of these groups would be randomly eliminated, but this never happened. The elimination procedure was followed until only one group remained. Within this group, the subject with the highest individual amount of EMUs received a payoff of $€ 30$. Again, if more subjects had the highest total, one of the subjects would be randomly picked as the winner, but this did not happen in our experiment. Thus, the number of competing groups decreases throughout the game, and only the individual with most EMUs in the last remaining group receives pay-off. We call this the "together alone" game, which is special because its tournament setting ensures the strongest possible conflict between individual interests in group benefits and personal benefits.

Given that a game starts with 12 subjects divided in 4 groups, the game can involve no more than three group eliminations. The three rounds followed by elimination were randomly selected and this happened to lead to a total of 9 rounds.

To study effects of learning within the context of the game rules imposed on the subjects, the game was played three times. This was done with the same 12 subjects, yet redistributed over groups such that each subject was never together with the same subject in a group in more than one game. The random selection of elimination rounds led to a total of 7 rounds in each of the two following games. Note that the subjects never had prior information on the number of rounds and the rounds followed by elimination.

Game execution. A total of 10 sessions were carried out using the computer facilities of CREED (Center for Experimental Economics and political Decision making) at the University of Amsterdam. The experimental setup was approved by the CREED department. Students were recruited using the database of CREED; participation was by informed consent. They received an e-mail announcing the upcoming experiment and could enrol online. Every participant was paid a show-up fee of $€ 10$ and only winners of a game were paid an additional $€ 30$. Students who did show up but could not participate were paid $€ 5$. A translation of the Dutch instructions into English is available as Supplementary Information. There were five sessions in the group competition treatment (i.e., ten replicate experiments), four sessions in the group-structured control treatment (i.e., eight replicate experiments) and one in the one-group control treatment. For this last session 21 students formed seven groups of three (i.e., seven replicate experiments). The other sessions were done with 24 students each split into two independent replicate groups of 12. Z-Tree (developed by Urs Fischbacher at the University of Zürich) was used to setup and run the experiment on computers.

Statistical analysis. To compare average investments among treatments involving group elimination (Fig. 1), we selected the per-replicate average investments in consecutive rounds only for those two groups per replicate that survived elimination events until the last round of the game. Thus, these data are independent of groups within replicate but represent repeated measures over rounds of a game. Data from groups that were eliminated during a game were not used for analysis. For the no-elimination treatment, the per-replicate average investments correspond to one group per replicate, as the participants had no information about other groups. To analyze a learning effect in individual investments over the three consecutive games (Fig. 2), we selected the per-individual investment only in round one of each game.

To approximate normality, we applied angular transformation (arcsin(square root)) to the average proportional investments per group. These data were analysed per game using repeated measures ANOVA (with treatment as categorical factor) with a Bonferroni post-hoc test. Caution should be exercised in the interpretation of this analysis because the variances among treatments are nonhomogeneous. Differences in average investment level among replicates of the lethal groupelimination treatment in the round before and the round after an elimination event were subjected to paired t-tests, again using the transformed data. A learning effect in the first-round investments from the first to the third game was inferred from an ANOVA applied to the transformed proportional investment data of subjects in the first round of each of the three games.

1. Fehr, E. \& Fischbacher, U. The nature of human altruism. Nature 425, 785-791 (2003).

2. Nowak, M. A. Five rules for the evolution of cooperation. Science 314, 1560-1563 (2006).

3. Bowles, S. \& Gintis, H. A Cooperative Species: Human Reciprocity and its Evolution. Princeton University Press: Princeton, NJ (2011).

4. Nowak, M., Tarnita, C. \& Wilson, E. O. The evolution of eusociality. Nature 466, 1057-1062 (2010).

5. Lion, S., Jansen, V. \& Day, T. Evolution in structured populations: beyond the kin versus group debate. TREE 26, 193-201 (2011).

6. Price, M. E. Group selection theories are now more sophisticated, but are they more predictive? Evol. Psy. 10, 45-49 (2012).

7. Hagen, E. H. \& Hammerstein, P. Game theory and human evolution: A critique of some recent interpretations of experimental games. Theor. Popul. Biol. 69, 339-348 (2006)

8. Sober, E. \& Wilson, D. S. Unto Others - The Evolution and Psychology of Unselfish Behavior. Cambridge, MA: Harvard University Press (1998). 
9. Boyd, R., Gintis, H., Bowles, S. \& Richerson, P. J. The evolution of altruistic punishment. Proc. Natl. Acad. Sci. USA 100, 3531-3535 (2003).

10. Henrich, J. Cultural group selection, coevolutionary processes and large-scale cooperation. J. Econ. Behav. Org. 53, 3-35 (2004).

11. Traulsen, A. \& Nowak, M. A. Evolution of cooperation by multilevel selection. Proc. Natl. Acad. Sci. USA 103, 10952-10955 (2006).

12. Bowles, S. Group competition, reproductive leveling, and the evolution of human altruism. Science 314, 1569-1572 (2006).

13. Bornstein, G. Intergroup conflict: individual, group, and collective interests. Pers. Soc. Psych. Rev. 7, 129-145 (2003).

14. Bornstein, G., Winter, E. \& Goren, H. Experimental study of repeated team-games. Eur. J. Polit. Econ. 12, 629-639 (1996).

15. Tan, J. \& Bolle, F. Team competition and the public goods game. Econ. Lett. 96, 133-139 (2007).

16. Puurtinen, M. \& Mappes, T. Between-group competition and human cooperation. Proc. R. Soc. B. 276, 355-360 (2009).

17. Ackermann, M. et al. Self-destructive cooperation mediated by phenotypic noise. Nature 454, 987-990 (2008).

18. Rockenbach, B. \& Miliski, M. The efficient interaction of indirect reciprocity and costly punishment. Nature 444, 718-723 (2006).

19. Ostrom, E. Governing the Commons. Cambridge (UK): Cambridge University Press (1990).

\section{Acknowledgements}

This paper is part of the research project "Simultaneous evolution of social norms and social behavior: a combined theoretical and experimental approach" funded by the Netherlands' Organisation for Scientific Research (NWO). We thank the Editorial Board Member, Shane Richards, for very thoughtful and pertinent comments on earlier versions of our manuscript.

\section{Author contributions}

M.E. designed experiments, analysed data and wrote the paper; R.K. and X.v.d.S. performed experiments; E.R. supervised experiments; and M.W.S. designed experiments and wrote the paper.

\section{Additional information}

Supplementary information accompanies this paper at http://www.nature.com/ scientificreports

Competing financial interests: The authors declare no competing financial interests.

License: This work is licensed under a Creative Commons

Attribution-NonCommercial-NoDerivs 3.0 Unported License. To view a copy of this license, visit http://creativecommons.org/licenses/by-nc-nd/3.0/

How to cite this article: Egas, M., Kats, R., van der Sar, X., Reuben, E. \& Sabelis, M.W Human cooperation by lethal group competition. Sci. Rep. 3, 1373; DOI:10.1038/srep01373 (2013). 